\title{
Factores que influyen en la recuperación funcional del paciente adulto mayor con fractura de cadera.
}

\section{Factors influencing in functional recovery of the elderly patient with hip fracture.}

Pág. 44,50

Recibido: 24-07-2020

Aceptado:27-08-2020

Licda. Marcela Villalobos Ulate ${ }^{1}$

1. Fisioterapeuta, Máster en rehabilitación de la persona adulta mayor, Docente de Fisioterapia, San José , Costa Rica.

\section{RESUMEN:}

Este artículo de revisión bibliográfica pretende identificar los factores que eventualmente pueden influir en la recuperación funcional de los pacientes adultos mayores, posterior a una fractura de cadera.

Se concluye que el proceso de recuperación funcional es multifactorial y puede verse influido positiva o negativamente por factores tales como la edad, el estado funcional previo, el estado nutricional, el estado cognitivo, la presencia de depresión, la presencia de falla cardiaca, medicamentos prescritos, el miedo a caer posterior a la fractura, y los roles que tiene la persona en la vida cotidiana.

\section{INTRODUCCIÓN}

Las fracturas de cadera generalmente ocurren en pacientes ancianos frágiles y se asocian con una importante morbimortalidad en el primer año. (Gamboa-Arango et al, 2020).

La coexistencia de comorbilidades y la disminución de la función cognitiva en el adulto mayor disminuyen la capacidad de respuesta del organismo y favorecen una situación de mayor fragilidad. Del mismo modo, el aumento del consumo de fármacos en esta población, especialmente los medicamentos psicotrópicos, alteran aún más estas capacidades, lo que facilita las caídas y, por ende, la aparición de la fractura. (Vento-Benel y Salinas-salas, 2017).

Las fracturas de cadera constituyen un importante problema de salud pública en la población adulta mayor, con repercusiones sanitarias (incremento de morbimortalidad y deterioro funcional), económicas y asistenciales (aumento del grado de dependencia e institucionalización), 
es también una entidad prevalente en un grupo etario de edad avanzada, y es una de las causas más frecuentes de ingreso hospitalario en personas de más de 65 años. (Vento-Benel y Salinas-Salas, 2017).

Se infiere entonces que, la fractura de cadera, por su alta morbimortalidad posterior al evento, dentro de la población adulto mayor, supone un problema de gran importancia asistencial, de gestión hospitalaria y de gasto económico y social en las próximas décadas. El objetivo tras el tratamiento es conseguir el nivel de independencia y de deambulación previo. Para ello no basta con un tratamiento médico y quirúrgico correcto, sino que precisarán de un tratamiento rehabilitador adecuado y de apoyo social, pues muchos de estos pacientes son incapaces de conseguirlo por ellos mismos. (Vento-Benel y Salinas-Salas, 2017).

\section{OBJETIVO GENERAL:}

Identificar los factores que influyen en la recuperación funcional del paciente adulto mayor, post fractura de cadera

\section{OBJETIVOS ESPECÍFICOS}

- Estudiar el proceso de recuperación funcional del paciente adulto mayor post fractura de cadera

- Analizar los aspectos que intervienen en el pronóstico de los pacientes post fractura de cadera

\section{METODOLOGÍA:}

Se realizó una búsqueda bibliográfica en las siguientes bases de datos: Science Direct, EBSCO y PUBMED en el periodo comprendido entre el 01 al 12 de julio de 2020, con las siguientes palabras clave: Fractura de cadera, adulto mayor, anciano, recuperación funcional, funcionalidad.

\section{CRITERIOS DE INCLUSIÓN:}

- Artículos científicos

- Artículos publicados en idioma inglés o español

- Publicados entre los años 2015 a 2020

\section{CRITERIOS DE EXCLUSIÓN:}

Después de leer el título y el resumen, se excluyen los artículos que no tienen relación con el tema de este artículo. Como resultado se procedió al análisis de 15 artículos en concordancia con el tema y objetivos de investigación.

\section{Discusión:}

De acuerdo con la investigación bibliográfica, son diversos los factores que pueden intervenir en la recuperación funcional de los pacientes adultos mayores con fractura de cadera.

Uno de los factores es la edad. Recientemente, en su estudio prospectivo, Gamboa Arango y colaboradores (2020), estudiaron a 271 pacientes mayores de 69 años, de los cuales $146(54.8 \%)$, mantuvieron la funcionalidad a los 12 meses y 122 (45.2\%) no. Entendiendo como mantenimiento funcional a aquellos pacientes que han perdido entre 0-15 puntos en el Índice de Barthel con respecto al anterior a la fractura. Los pacientes que mantienen el estado funcional son más jóvenes, con mejores puntajes en los índices de Lawton antes de la fractura y Barthel al alta, de manera que concluyeron que los factores pronósticos del mantenimiento funcional a los 12 meses son la edad y la capacidad de realizar actividades instrumentales de la vida diaria. 
Asociado también a la edad, Benchimol y colaboradores (2020), a través de una cohorte prospectiva que incluyó 952 pacientes con fractura de cadera clasificados en pacientes de edad avanzada (EP $=<65$ y $<85$ años), de los cuales el $43 \%$ eran EP y el $57 \%$ eran VEP, concluyeron que los VEP fueron más dependientes y más débiles.

Los resultados esperables en el incremento de la funcionalidad, luego de un proceso de rehabilitación también pueden verse mediados por la presencia de síntomas depresivos en la persona mayor, especialmente en actividades que implican movilidad. En personas con fractura de cadera, los síntomas depresivos moderados a severos se relacionan a una menor recuperación en la capacidad de caminar, institucionalización o muerte, luego de un proceso rehabilitador después de 1 año (Aravena, 2017). Este autor, en un estudio de cohorte que incluyó 59 personas mayores con fractura de cadera y deterioro cognitivo seguidas durante su proceso de rehabilitación, determinó que la mayor presencia de síntomas depresivos se correlacionó con menores resultados en la eficiencia del FIM motor, mayor estancia hospitalaria, y a una menor participación en el tratamiento. (Aravena, 2017). En esto coinciden Rathbun y colabores (2018), quienes mencionan que la presencia de síntomas depresivos durante los primeros 6 mes después de una fractura de cadera tiene un impacto significativo en la recuperación funcional.

El uso de ayudas técnicas también es un aspecto a considerar dentro de la rehabilitación funcional de un adulto mayor con depresión. Las personas mayores depresivas utilizan con mayor frecuencia dispositivos de ayuda (adaptaciones en el hogar, bastones, entre otros) dado que presentan un incremento en su dependencia, en comparación a población sin síntomas anímicos. (Aravena, 2017) ,lo cual también influye en la recuperación e independencia funcional.

Por otra parte, laboni y colaboradores (2017), en su estudio longitudinal prospectivo, incluyeron a 477 adultos mayores de 60 años sometidos a reparación quirúrgica de fractura de cadera, que no se presentaban libres de delirio, demencia o depresión e indicaron que los adultos mayores con fractura de cadera tienen un riesgo mayor de prescripción de medicamentos potencialmente inapropiados $y$, pueden ser particularmente vulnerables a sus efectos adversos, concluyendo que el uso de medicación potencialmente inapropiada se asocia con un tiempo mayor de recuperación funcional en adultos mayores que fueron sometidos a una cirugía por fractura de cadera, particularmente aquellos que usan dos o más medicamentos de forma inapropiada.

En lo que se refiere a la influencia del estado cognitivo, según un estudio observacional retrospectivo que incluyó 1258 pacientes con edades superiores a 69 años y que sufrieron fractura de cadera, a los cuales se les dió seguimiento durante 12 meses, Tazarona y colaboradores (2015), compararon pacientes con demencia y pacientes con estado cognitivo preservado. Se concluyó que, los pacientes con demencia tuvieron menor probabilidad de recuperación funcional y mayor probabilidad de morir después del egreso hospitalario.

En otro estudio prospectivo con 359 pacientes de 75 años en adelante, de Miguel y colaboradores (2018), determinaron que el pronóstico de recuperación funcional depende de la edad, estado funcional previo, antecedentes clínicos de demencia y la presencia de delirium durante la estancia hospitalaria. 
También, Uriz y colaboradores (2015), estudiaron a 314 pacientes mayores de 65 años admitidos en rehabilitación después de una cirugía por fractura de cadera y, encontraron que la capacidad previa de caminar y la presencia de complicaciones, tales como úlceras por presión y delirium, juegan un rol más importante que el estado cognitivo en la recuperación de estos pacientes.

Porsuparte,ParejaSierray colaboradores(2017), realizaron un estudio prospectivo de un grupo de 130 pacientes mayores de 75 años de edad con fractura de cadera osteoporótica y, determinaron que los pacientes con gran discapacidad física, deterioro cognitivo severo e institucionalizados en asilos de ancianos antes de la fractura tienen una peor recuperación funcional después de la cirugía. Estos autores también señalaron que la prescripción de suplementos nutricionales a pacientes con indicación permitida, mejoraba su recuperación física después de la fractura de cadera y resaltaron que la evaluación del estado físico, cognitivo y nutricional previos a la fractura deberían ser la base del plan de tratamiento individual, ya que tienen un gran valor pronóstico.

Otros autores también asocian el estado nutricional al estado funcional en pacientes con fracturas de cadera. Así, por ejemplo, Caicedo y colaboradores (2019) mencionan en su estudio de 177 ancianos con fractura de cadera, a los cuales se les analizó el estado funcional y nutricional dos semanas previo al evento de fractura, que el $45.76 \%$ eran independientes para sus actividades básicas de la vida diaria y $54.23 \%$ presentaban algún grado de deterioro funcional. De los pacientes con algún grado de malnutrición, solo $32.81 \%$ eran independientes funcionalmente y $79.59 \%$ con adecuado estado nutricional eran independientes. Concluyendo así que, el estado nutricional y funcional son factores asociados a presentar fractura de cadera.

Malafarina y colaboradores (2018), no solamente mencionan que la malnutrición es prevalente en pacientes con fractura de cadera, sino que esto no siempre es reconocido, y señalan que los bajos índices antropométricos están asociados a una alta prevalencia de complicaciones durante la hospitalización y una peor recuperación funcional. Incluso, la malnutrición está asociada a un incremento de la mortalidad, por lo cual, una intervención nutricional se considera efectiva en el mejoramiento del estado nutricional, el desempeño físico y una mayor recuperación funcional posterior a una fractura de cadera.

Itagaki y colaboradores (2019), por su parte, señalan la falla cardiaca como un factor de riesgo en la disminución de la función física y caídas. Por lo que buscaron examinar si esta afecta la recuperación después de una fractura de cadera. Realizaron un estudio que incluyó a 122 pacientes con fractura de cadera a quienes dividieron en pacientes con y sin falla cardiaca y concluyeron que esta condición sí afecta la recuperación funcional después de la fractura de cadera, independientemente de la condición física previa al evento de fractura y, sugieren que se requiere mayor investigación en pacientes con ambas condiciones para desarrollar estrategias que permitan superar la pobre recuperación funcional observada en estos pacientes.

De acuerdo con Bower y colaboradores (2016), el miedo a caer también es común después de una fractura de cadera y está asociado a la recuperación funcional. Ellos realizaron un estudio prospectivo que incluyó 241 
participantes, conginitivamente intactos, de 60 años en adelante que requirieron reparación quirúrgica de fractura de cadera, concluyendo que este temor miedo a caer está asociado con una pobre recuperación funcional 1 año después de la fractura y, es un problema modificable que representa potencial objetivo de intervención para mejorar los resultados funcionales después de una fractura de cadera

Algo interesante de agregar es que según Nardi y colaboradores (2018), el rol que desempeña la persona puede influir en su recuperación. Ellos realizaron un estudio prospectivo observacional para evaluar si los adultos mayores con un rol de cuidador tenían mejor recuperación funcional después de una fractura de cadera que quienes no desempeñan este tipo de roles. Este estudio incluyó 107 pacientes con fractura de cadera y edades superiores a 65 años a quienes se les consultó si estaban al cuidado de otra persona, una mascota o plantas, determinándose que aquellos que tenía un rol de cuidador de cualquier clase, especialmente de plantas, tenían una mejor recuperación a corto plazo después de la fractura de cadera. $Y$ aquellos que desempeñaban un rol como cuidadores de otras personas parecían mostrar beneficio en la recuperación a largo plazo.

Conclusiones:

Se concluye que la recuperación funcional en los pacientes adultos mayores, posterior a una fractura de cadera, puede verse influenciada de manera multifactorial.

Factores tales como la edad, estado nutricional, estado funcional previo, medicamentos prescritos, presencia de falla cardiaca, alteraciones cognitivas, presencia de depresión, temor a caer, e incluso los roles adquiridos por el paciente antes de la fractura, inciden directamente en el pronóstico que tenga el paciente adulto mayor, posterior a la fractura de cadera y en la evolución que siga durante su proceso de recuperación.

Considerando que varios de ellos pueden ser modificados, es importante intervenir sobre ellos de manera preventiva, ya que esto podría mejorar el pronóstico y la recuperación funcional de los pacientes adultos mayores, en caso de que ocurra una fractura de cadera.

\section{REFERENCIAS}

Aravena, José M. (2017). Comprendiendo el impacto de los síntomas depresivos en la funcionalidad de las personas mayores. Revista chilena de neuro-psiquiatría, 55(4), 255-265. https://dx.doi. org/10.4067/s0717-92272017000400255

Benchimol, J. A., Elizondo, C. M., Giunta, D. H., Schapira, M. C., Pollan, J. A., Barla, J. D., Carabelli, G. S., \& Boietti, B. R. (2020). Survival and functionality in the elderly over 85 years of age with hip fracture. Revista Española de Cirugía Ortopédica y Traumatología. https://doi.org.ezproxy.ucimed. com/10.1016/j.recot.2020.02.001 
Bower, E. S., Wetherell, J. L., Petkus, A. J., Rawson, K. S., \& Lenze, E. J. (2016). Fear of Falling after Hip Fracture: Prevalence, Course, and Relationship with One-Year Functional Recovery. The American journal of geriatric psychiatry: official journal of the American Association for Geriatric Psychiatry, 24(12), 1228-1236. https://doi.org/10.1016/j.jagp.2016.08.006

Caicedo-Correa, S. M., Aruachan-Torres, S. A, Segura-Valencia, A. I, Chavarro-Carvajal, D. A. (2019). Asociación del estado nutricional y funcional en ancianos con fractura de cadera. Acta Medica colombiana, 44(1), 7-10. https://doi.org.ezproxy.ucimed.com/10.36104/amc.2019.1035

de Miguel Artal, M., Roca Chacón, O., Martínez-Alonso, M., Serrano Godoy, M., Mas Atance, J., \& García Gutiérrez, R. (2018). Fractura de cadera en el paciente anciano: factores pronósticos de mortalidad y recuperación funcional al año [Hip fracture in the elderly patient: Prognostic factors for mortality and functional recovery at one year]. Revista espanola de geriatria y gerontologia, 53(5), 247-254. https://doi.org/10.1016/j.regg.2018.04.447

Gamboa-Arango, A., Duaso, E., Formiga, F., Marimón, P., Sandiumenge, M., Salgado, M. T., Escalante, E., Lumbreras, C., \& Tarrida, A. (2020). Pronostic factors of good functionality at 12 months of a hip fracture. Maluc Anoia study. Revista Espanola de Cirugía Ortopédica y Traumatología, 64(1), 57-63. https://doi.org.ezproxy.ucimed.com/10.1016/j.recot.2019.06.007

laboni, A., Rawson, K., Burkett, C., Lenze, E. J., \& Flint, A. J. (2017). Potentially Inappropriate Medications and the Time to Full Functional Recovery After Hip Fracture. Drugs \& aging, 34(9), 723-728. https://doi.org/10.1007/s40266-017-0482-6

Itagaki, A., Kakizaki, A., Funahashi, M., Sato, K., Yasuhara, K., \& Ishikawa, A. (2019). Impact of heart failure on functional recovery after hip fracture. Journal of physical therapy science, 31(3), 277-281. https://doi.org/10.1589/jpts.31.277

Malafarina, V., Reginster, J. Y., Cabrerizo, S., Bruyère, O., Kanis, J. A., Martinez, J. A., \& Zulet, M. A. (2018). Nutritional Status and Nutritional Treatment Are Related to Outcomes and Mortality in Older Adults with Hip Fracture. Nutrients, 10(5), 555. https://doi.org/10.3390/nu10050555

Nardi, M., ... y Bischoff-Ferrari, H. A. (2018). Association between Caregiver Role and Short- and Long-Term Functional Recovery after Hip Fracture: A Prospective Study. Journal of the American Medical Directors Association, 19(2), 122-129. https://doi.org/10.1016/j.jamda.2017.08.009

Pareja Sierra, T., Bartolomé Martín, I., Rodríguez Solís, J., Bárcena Goitiandia, L., Torralba González de Suso, M., Morales Sanz, M. D., \& Hornillos Calvo, M. (2017). Predictive factors of hospital stay, mortality and functional recovery after surgery for hip fracture in elderly patients. Factores determinantes de estancia hospitalaria, mortalidad y evolución funcional tras cirugía por fractura de cadera en el anciano. Revista espanola de cirugía ortopédica y traumatología, 61(6), 427-435. 
https://doi.org/10.1016/j.recot.2017.06.002

Rathbun, A. M., Shardell, M. D., Stuart, E. A., Gruber-Baldini, A. L., Orwig, D., Ostir, G. V., Hicks, G. E., Hochberg, M. C., \& Magaziner, J. (2018). Persistence of depressive symptoms and gait speed recovery in older adults after hip fracture. International journal of geriatric psychiatry, 33(7), 875-882. https://doi.org/10.1002/gps.4864

Tarazona-Santabalbina, F. J., ......y Avellana Zaragoza, J. A. (2015). Severity of cognitive impairment as a prognostic factor for mortality and functional recovery of geriatric patients with hip fracture. Geriatrics \& gerontology international, 15(3), 289-295. https://doi.org/10.1111/ggi.12271

Uriz-Otano, F., Uriz-Otano, J. I., \& Malafarina, V. (2015). Factors associated with short-term functional recovery in elderly people with a hip fracture. Influence of cognitive impairment. Journal of the American Medical Directors Association, 16(3), 215-220. https://doi.org/10.1016/j.jamda.2014.09.009 Vento-Benel, F.C., Salinas-Salas. C. R. (2017) Fractura de cadera en el adulto mayor. [Cartas al Editor]. Rev. Fac. Med. Hum. 17(2):112. https://doi.org/10.25176/RFMH.v17.n2.845 